\title{
La convergencia y la sinergia en las micro-finanzas y su accesibilidad para los emprendedores autónomos
}

\section{Convergence and synergy in microfinance and its accessibility for self-employed entrepreneurs}

\author{
Mancero Mosquera, Jacinto Humberto*1 y Carrillo Mañay, Venustiano ${ }^{1}$ \\ ${ }^{1}$ Universidad Católica Santiago de Guayaquil \\ *humbertomancero@hotmail.com
}

\begin{abstract}
Resumen
Se pretende aporta en la solución al problema de porque los emprendedores autónomos de Guayaquil - Ecuador no lograr acceder a los beneficios de las micro-finanzas de forma eficiente y siguen dependiendo de la usura como su principal proveedor de microcrédito. El propósito es diseñar un procedimiento integral que de forma convergente logre la sinergia para lograr prosperidad sostenible y equidad social. La proposición se sustenta en un análisis profundo de las teorías existen y desarrolladas por diferentes autores e investigadores desde el origen de las finanzas, el trabajo autónomo y emprendimiento autónomo, orientados a los niveles de informalidad denominada como trabajo por cuenta- propia, que responde a mejorar los niveles de sub-empleo y desempleo social, siendo la pobreza la mayor de las calamidades, común a todos los países y sistemas económicos, políticos y sociales existes y actuales. La metodología de investigación de tipo teórico mixta, histórica, descriptiva, comparativa porque analiza documentos existentes, se realiza una revisión de la literatura de los conceptos relacionados a la accesibilidad que deben tener los micro-emprendedores autónomos. Además se busca la integración de diferentes actores sociales con herramientas y metodologías operativas de trabajo para consolidar en una "Red de servicios integrados al micro-emprendedor autónomo" con todos los actores sociales, comprometidos en atender y ayudar en la solución o al menos reducir al mínimo la inequidades y exclusión existentes donde los pobres sean realmente incluidos en los procesos de micro-finanzas como herramienta para vencer la pobreza y dignificar sus emprendimientos y desarrollarlos a la prosperidad en el largo plazo.
\end{abstract}

Palabras clave: Emprendedores ambulantes, trabajo por cuenta propia, micro-emprendimientos autónomos, micro-finanzas integral..

\begin{abstract}
It seeks to contribute to the solution to the problem of the fact that the autonomous entrepreneurs of Guayaquil - Ecuador fail to access the benefits of microfinance efficiently and continue to depend on usury as their main provider of microcredit. The intention is to design an integral process that converges synergistically to achieve sustainable prosperity and social equity. The proposal is based on an in-depth analysis of the theories that exist and are developed by different authors and researchers from the origin of finance, autonomous work and autonomous entrepreneurship, oriented to levels of informality called self-employment, which responds to improving levels of underemployment and social unemployment, with poverty being the greatest of calamities, common to all countries and existing economic, political and social systems. The research methodology of mixed theoretical, historical, descriptive, comparative because it analyzes existing documents, a review of the literature of the concepts related to the accessibility that the autonomous micro-entrepreneurs must have. In addition, it seeks the integration of different social actors with operational tools and methodologies to consolidate in a "Network of integrated services to the autonomous micro-entrepreneur" with all social actors, committed to attend and help in the solution or at least reduce to minimum the existing inequities and exclusion where the poor are actually included in microfinance processes as a tool to overcome poverty and dignify their enterprises and develop them to prosperity in the long term.
\end{abstract}

Key words: Entrepreneurs traveling, self-employment, autonomous micro-enterprises, integral microfinance..

\section{Introducción}

Las micro-finanzas son las herramientas más populares en la actualidad para acercarse como mecanismo de apoyo a los trabajadores informales, pobres, microempre- sarios, cuenta propistas, buhoneros, trabajadores autónomos, micro-emprendedores, vendedores ambulantes, nombres con los que se identifican a estos ciudadanos de escasos recursos que trabajan en las calles de las ciudades ofrecien- 
do: productos artesanales, artículos de dudosa procedencia y en especial alimentos y bebidas.

El natural financista de estos trabajadores lo constituyen los denominados chulqueros o usureros, que son banqueros de primera mano a que acceden este grupo social excluido tradicionalmente por la banca formal, desde su origen hasta los años 70's, cuando surgieron las novedosas teorías de las micro-finanzas, mediante los aporte del Ec. Mohamend Yunus ${ }^{1}$, padre del modelo de microcréditos, en la modalidad de grupos solidarios, en sus inicios para mujeres en su natal Bangladesh.

El sistema financiero tradicional dentro de las causas de porque no lograron atender a los clientes pobres y excluidos de la sociedad, desde el criterio puramente técnico de banquero ha sido muy bien identificado, con la siguiente aportación: Los bancos y otras instituciones financieras no han logrado satisfacer las necesidades de financiación de los pobres a nivel mundial. Las razones para el abandono a este grupo se encuentran en la naturaleza de las prácticas bancarias tradicionales, las instituciones financieras se basan en la aplicación de procedimientos ortodoxos como: la exigencia indispensable de garantías reales y la buena reputación de sus clientes originada en la experiencia crediticia y el adecuado uso de los distintos instrumentos de servicios financieros actuales como: el uso de chequeras, cultura de ahorro comprobable, la acumulación de bienes, pertenecer a la sociedad formal y vivir dentro de los perímetros formales y seguros de los territorios sean estos urbanos o rurales preferentemente.

Planteamiento del problema:

Siendo los microcréditos una extensión de la línea de negocio bancario tradicional, al homologarse los modelos de evaluaciones ortodoxos, garantías, calificación, para los pobres e informales, se volvieron de difícil acceso, por estar muy cercanos a la formalidad, los requisitos se convirtieron en las barreras iniciales para su fracaso inicial, era como decir te doy crédito pero como no califican con los requisitos exigidos, no se los puede ayudar y nuevamente la accesibilidad se volvió un mito o casi privilegio de excepción, a quienes ya estaban mejor establecidos, tenían más experiencia en negocios, más educación y establecidos en lugares fijos de trabajo.

Objetivo General:

Diseñar de forma integrada el procedimiento que facilite la accesibilidad a los emprendedores autónomos en su acceso a las micro-finanzas, para contribuir a su desarrollo de forma convergente, logrando la sinergia que los ayude a salir del círculo perverso de la miseria y pobreza, llevándolos al círculo de la prosperidad sostenible en el largo plazo.

Marco Teórico o antecedente:

La informalidad es un fenómeno de creciente complejidad. Detrás de ello, se esconden problemáticas estructu-

\footnotetext{
${ }^{1}$ Muhammed Yunus, jefe del programa de Economía Rural de la Universidad de Chittagong, dirigió un proyecto de investigación en el que se le ocurrió diseñar un sistema y metodología de crédito cuyo fin era la cobertura crediticia en zonas rurales, zonas donde no llegaban los servicios bancarios tradicionales. Premio Nobel de la Paz 2006
}

rales como la pobreza, la exclusión social, el desempleo, sub-empleo, inequidad social. La informalidad no puede ser analizada de manera aislada, sino que debe ser estudiada como parte de un todo, su tejido vinculante al trabajo dentro de un sistema económico. Este trabajo, más se ha focalizado a la falta de tributación, que es solo una arista del conjunto. (Serrano Mancilla, 2010)

Los informales o ambulantes, son las personas que trabajan y comercializan en las vías públicas, apareciendo determinados días y volviendo cíclicamente a poner sus puestos de venta en partes donde, originalmente, no fueron destinados. (Contreras Soto, 2007)

Según datos proporcionados por el Sistema de Rentas Internas SRI (Gráfico 1), al año 2013 el Ecuador tiene un $74,90 \%$ de sector informal, con relación a los demás países de América Latina, lo cual preocupa altamente, pues si bien es cierto incrementan el PIB pues aportan a la economía nacional, también generan irregularidad en el sistema comercial al competir deslealmente con el comercio formal, de allí la necesidad de políticas regulatorias y de control, para que las condiciones de competitividad seas más segmentadas en sus mercados.

Las micro-finanzas es la intermediación financiera a nivel local, es decir, se incluye no sólo los créditos sino los ahorros, los depósitos y otros servicios financieros. Es un procedimiento local, basado en instituciones locales que recogen recursos locales y los reasignan localmente. Además, se refieren a la provisión de servicios financieros como préstamos, ahorro, seguros o transferencias a hogares con bajos ingresos. La mayoría de las instituciones del sector se han dedicado al microcrédito.

Entre las principales características que tienen los programas de microcrédito podemos destacar las siguientes: Reducido monto a prestar. Normalmente, la cantidad prestada a través del microcrédito suele oscilar entre $\$ 100$ y $\$ 600$. Los plazos inferiores a un año. Las recuperaciones se cobran cuotas, semanales. Las actividades son las microempresas o los micro-negocios. Los microcréditos están dirigidos a los sectores más pobres de la sociedad y su objetivo final es el de generar una fuente regular de ingresos. (Lacalle Calderón, 2001).

Harvard estudió como caso a BancoSol de Bolivia por ser pionera en esta industria y en el año 2013, sucedió uno de los acontecimientos temidos. El Gobierno aprobó la ley 393 de Servicios Financieros, con enormes implicaciones para la institución, el $60 \%$ de sus préstamos se deben prestar al 11,5\% como tasa máxima. ¿Es eso factible?' El caso indica que $18 \%$ ya era una tasa baja, ¿Van a poder?”, explica Kurt Koenigsfest Gerente General. Harvard explica que: los microcréditos se otorgan en montos pequeños de 500, 800, 1.000 dólares, a corto plazo. Bolivia ya tenía una tasa de interés del $18 \%$, la más baja de la región y tal vez del mundo. En la región, Perú tiene una tasa de casi $30 \%$ para los microcréditos, México de $80 \%$ (esto porque los bancos prestan 10 dólares a una semana de plazo, además lo hacen en el campo, en poblaciones muy lejanas), Colombia 


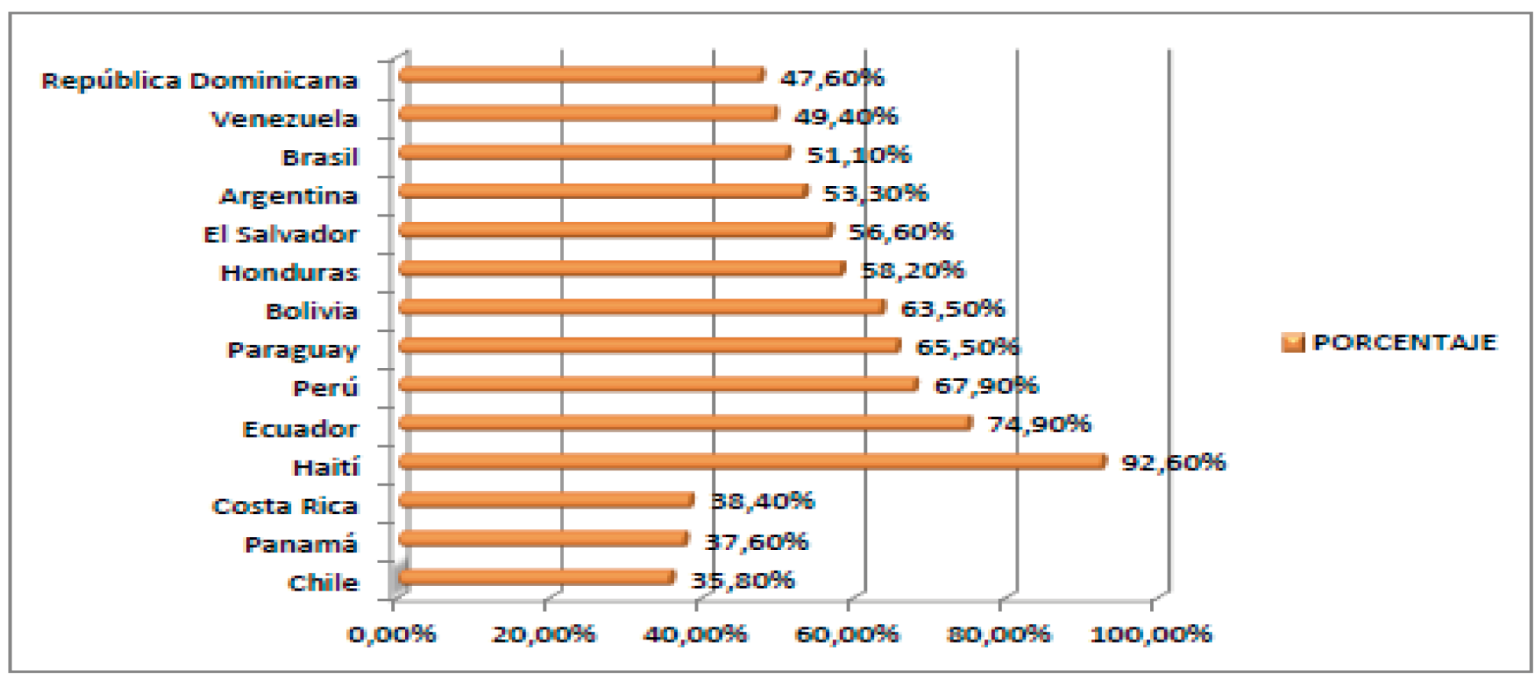

Figura 1. El comercio informal de américa y sus países más representativos, fuera de EUA., Canadá y México.

Fuente: SRI Servicio de Rentas Internas

de $30 \%$, y Ecuador de $30 \%$, por citar ejemplos referentes. (Natalí Vargas, 2016)

La microempresa en Bolivia es la respuesta creativa a las oportunidades del mercado frente a deficiencias estructurales de las economías en desarrollo o en transformación. En Bolivia, para más de la mitad de la población económicamente activa la posibilidad de trabajo depende de su propia capacidad para aprovechar las oportunidades que ofrece el mercado de bienes y servicios. BancoSol apoya la capacidad de los micros y pequeños empresarios de manera que éstos puedan desarrollar en forma más rápida sus negocios y así mejorar sus condiciones de vida. Los clientes del Banco en su mayoría son personas jóvenes, el $40 \%$ están entre los 35 y 45 años. El $46 \%$ son mujeres, por lo general provenientes de familias numerosas, con bajo nivel de educación.

Se toma como referente a esta institución líder y ejemplo claro de lo que son las micro-finanzas, en su conjunto y el gran aporte que tienen en un país como Bolivia, donde existe concentración de población indígena y pobreza. Casi la mitad de los clientes tienen tierras en el área rural, además de su domicilio en centros urbanos. Son personas que valoran mucho el esfuerzo propio y perciben al trabajo como única fuente para mejorar y progresar en la vida.

Dentro de los productos y servicios de micro-finanzas ofertadas por esta institución están los siguientes: 1) Fondo de garantía de créditos de vivienda de interés social; 2) Fondo de garantía de créditos para el sector productivo; 3) Microcréditos: Sol-oportuno, sol-vivienda social, solDPF, sol-productivo, sol-individual, sol-agropecuario, solvivienda, sol-efectivo, sol-vehículo; 4) Micro-seguros: solprotección, sol-protección/plus, sol-seguro de tarjeta de débito, 5) Giros y transferencias: giros-nacionales, girosinternacionales; 6) Micro-ahorros: Solecito de 0-12 años, sol-generación de 13-17 años, cuenta de ahorro, cuenta mayor, Depósitos a plazos; 7) Servicios: APP-Sol, InfoSolSMS, Sol-amigo, Tarjeta de débito, Servi-Sol, Agenciamóvil, Pago de Impuestos, Boleta de garantía, Sol-net, $\mathrm{ACH}$.

Surge como respuesta directa a la exclusión y marginación a los pobres del mundo de parte del sistema financiero formal, que solo se concentra en los niveles de Clase Media y alta y Corporativo pudiendo llegar a las microempresas, muy bien establecidas como excepción. (Ver Figura 2). "Las micro-finanzas se consideran como la herramienta más prometedora y menos costosa de la lucha contra la pobreza mundial."

Limitaciones de la investigación:

Los estudios serios que realizan las instituciones globales y mundiales, que se dedican a estudiar la exclusión social, pobreza, afectación de derechos humanos, inequidad y desigualdad social, representados por organizaciones como: ONU, FIDE, BM, BID, CEPAL, CUMBRE DE MICRO-FINANZAS, CGAP. Se realizan anual o cada dos años a manera de informes complementarios por países en funciones de indicadores sociales oficiales expresados en sus estadísticas públicas. Que son aproximaciones superficiales de las realidades sociales y económicas.

La investigación real a mayor nivel de profundidad debido a los altos costos que ésta representan se hacen cada diez años aproximadamente, siguiendo la línea de los censos poblacionales y económicos realizados en los países. Situación que genera una brecha en la calidad de la información de bases de datos confiables, oficiales a utilizar como referentes para la investigación, al contar con bases de datos, fuentes primarias y estadísticas analizadas y procesadas que sirven de punto de partida para estudios específicos.

De allí que cuando se requiere investigar más específicamente sobre estos temas las únicas opciones reales 
disponibles son la combinación y optimización de las necesidades que tienen los estudiantes de las carreras de grado y post-grado, quienes tienen la imperiosa necesidad de graduarse, siendo esta la oportunidad que disponen los docentes-investigadores, para ayudarse a realizar los estudios de campo puntuales a profundidad, hasta donde sea posible avanzar y obtener información más cercana a las realidades actuales que se viven en la sociedad, motivo de la investigación.

\section{Metodología}

La metodología es mixta, porque combina la revisión de la literatura existen con el tema de investigación, analiza, su evolución histórica, desde sus orígenes a la fecha en que se encuentran trabajos realizados, la etnografía aporta las vivencias que se tabulan en las entrevistas y sus historias de éxito y fracaso reales ocurridas a los micro-emprendedores ambulantes, que al final son los héroes anónimos, al subsistir con el desafío por estar con un pie en la legalidad y el otro en la ilegalidad, un poco forzados por esa falta de accesibilidad a las micro-finanzas, y la falta de comprensión de lo que deben ser. Y no considerárselas, tan sólo como sinónimo de microcréditos.

\section{Resultados}

Las fuentes de autofinanciamiento desarrolladas por los pobres, microempresarios o emprendedores autónomos alrededor del mundo:

EN investigaciones realizadas se encuentran similitud en las sociedades de África, Latinoamérica, Centroamérica y Europa con mínimas variaciones de como satisfacer las necesidades de financiamiento, mediante los siguientes mecanismos:

1) Ayuda de familiares, amigos y vecinos

2) Grupos de autoayuda en sus varias modalidades

- Rotating Savings and Credit Associations ROSCA y su variedad de nombres

- Asociación de créditos y ahorros acumulados ACAA o Accumulating Savings and Credt Associations (ASCA)

- Grupos de autoayuda o Self Help Groups (SHG);

3) Otras prácticas de financiamiento

- Las mercaderías en consignación

- Financiamiento al partido

4) Prestamistas

- Las instituciones formales u oficiales

- Prestamistas usureros o chulqueros

5) Instituciones públicas y privadas de ayuda

- Las organizaciones no gubernamentales (ONG's)

\section{Familiares, amigos y vecinos:}

Sustentados en la solidaridad y ayuda mutua, esta es la primera fuente de financiamientos natural existente, de fondos de bajo monto debido a la flexibilidad de las condiciones y en muchos casos esta excepta del pago de interés, garantías, siendo tolerante en los plazos pactados inicialmente. Este es mecanismos que utilizan los migrantes de países sub-desarrollados para viajar en busca del sueño norteamericano o europeo (España e Italia) en sus mejores momentos de auge económico.

II. Grupos de autoayuda en sus varias modalidades o Self Help Groups (SHG):

Grupos formados por miembros de una comunidad cercana y conocida entre sí, que se reúnen para potencializar sus ahorros personales pequeños, mediante la reunión de cuotas iguales de los miembros participantes. Estos grupos originarios de África y Asia, existiendo versiones en Latinoamérica se los conoce con varios nombres:

a) Rotating Savings and Credit Associations ROSCA/ Tontines/ Sunsun/ Cadena/ Argolla/ Rueda/ Circulo; Club de compra; Consorcios.

La ROSCA es un sistema exitoso de alta participación en diferentes áreas geográficas como: Asia, África y Latinoamérica, mostrando que en la práctica es un excelente instrumento mixto de ahorro y crédito. Así, algunos estudios muestran que participaban en este sistema informal el $40 \%$ de los clientes de Bank Rakyat Indonesia (BRI), una de las mayores Instituciones Micro-financieras del mundo, cuyo monto ha llegado a representar el $20 \%$ de los depósitos bancarios totales en sus fuentes de captaciones de recursos monetarios.

La operatividad de esta modalidad en su versión original es sencilla: consisten en formar un grupo de personas cercanas y conocidas, que realizan aportes iguales en un mismo periodo para formar un "fondo común o bolsa", que se asigna a uno de sus integrantes, mediante sorteo y así queda establecida la fecha en que cada integrante recibirá su fondo, hasta liquidar el grupo total sin faltar nadie en recibir la bolsa reunida de los aportes. Existe la opción que después de la asignación de forma personal alguien negocie o se haga el favor entre sí de ceder su turno de forma solidaria, o el grupo por consenso decida ayudar a un integrante, que presente alguna necesidad emergente de excepción. En su forma más simple, este sistema de autoayuda, no cobra ni pagan intereses, solo se potencializa el ahorro grupal en periodos no mayores a un año. Normalmente el promotor se constituye en el administrador del proceso.

b) Asociación de créditos y ahorros acumulados ACAA o Accumulating Savings and Credt Associations (ASCA):

Es una evolución domestica de la ROSCA que se forma creando un fondo común con los aportes en partes iguales de sus participantes. Su asignación se hace en partes a varios miembros el grupo, cobrando una tasa de interés más baja que el mercado referencial la misma que va formando un patrimonio-capital de todos. Por excepción se pueden conceder estos préstamos a no miembros de grupo conocidos y recomendados por los integrantes que lo aprueban, se les reconoce una tasa de interés a quienes no usan el dinero y tienen saldo a favor a una tasa más baja 
que el mercado igual equivalente para apalancar el proceso de generación de ganancia dejando el spread que constituye la diferencia entre la tasa activa de colocación-préstamo y la tasa pasiva de captación-ahorro y se pagan intereses a quienes tienen saldos acreedores, mecanismos que se la considera como la antesala de las cooperativas de ahorro y crédito.

c) Grupos de autoayuda o Self Help Groups (SHG):

En la línea de potencializar el ahorro, la India aporta el modelo de auto-ayuda similar al ASCA, que se diferencia del mismo en el menor número de integrantes de los grupos de 10 a 20 personas (preferiblemente mujeres), que hacen aportaciones periódicas para constituir un fondo común, que solo se utiliza para casos de emergencia u otra prioridad. Estos fondos en muchos casos son obtenidos de préstamos del sistema formal, al que pagan los intereses y conservan el capital para aportarlo al grupo aumentando su monto. (Nair, 2005).

Los grupos de autoayuda en la India, son casos exitosos referentes históricos. Es otra de las metodologías organizadas más extendidas. Su crecimiento se ha favorecido por el programa gubernamental, que aporta fondos propios y capta recursos de instituciones multilaterales externas, que buscan vincular a estos grupos con instituciones formales que los financien. El programa del Banco Nacional de Agricultura y Desarrollo Rural (NABARD) logró que, entre 1992 al 2003, se formaron 700.000 grupos que recibieron 425 millones de dólares externamente, impactando a 10 millones de personas (Nair, 2005), estas cifras, se han incrementado a 15 millones de personas en el 2007.

\section{Otras prácticas de financiamiento:}

a) Las mercaderías en consignación:

Los propietarios de las mercaderías las entregan a los comerciantes, para que las comercialicen a cambio una comisión o diferencial de precio, con la ventaja de no tener que adquirir las mismas, ni endeudarse; trabajan con el capital del propietario de la mercadería, modalidad muy popular en los emprendimientos de ropa, perfumes, joyas de fantasía, realizados por personas que combinan su empleo en relación de dependencia con el emprendimientoambulante-autónomo, realizado también en su hogar y otras empresas donde tienen amigos o generan una cartera de clientes mediante el sistema de referidos. Normalmente desarrollados en los sectores urbanos u otras zonas rurales cercanas.

b) Financiamiento al partido:

Esta modalidad de financiamiento se genera mediante los créditos que otorgan financistas informales del sector agrícola, para financiar las cosechas: agrícolas de ciclo corto y de pequeña escala, avícolas, acuícolas, piscícola, para consumo interno, "caso ecuatoriano". Siendo esta modalidad, la antesala. De los "Contratos de fideicomiso" que se utilizan para garantizar la provisión de materias primas, mediante el financiamiento en el proceso productivo y abastecerse de insumos para procesar o vender directamente a los fabricantes e intermediarios.

\section{Prestamistas:}

Según el estudio realizado por: (Banerjee \& Duflo 2007) muestra que la necesidad de financiamiento informal es más frecuente y habitual entre lo población pobre, que entre la clase media y alta formal. En el mercado en general existen dos clases de prestamistas:

a) Las Instituciones formales u oficiales:

Por lo general son las instituciones que trabajan dentro de las regulaciones legales, representados por las Instituciones de micro-finanzas (IMF's) especializadas y las extensiones de línea de negocios de los bancos formales establecidos; que participan de esta actividad en varios casos obligados por las nuevas regulaciones vigentes, que les exigen apoyar a los trabajadores informales o las organizaciones que comprendieron que este sector económico, es una actividad con alto componente de rentabilidad social y simultáneamente financiera, que ayuda a la generación de más utilidades para sus accionistas.

\section{b) Prestamistas usureros o chulqueros:}

Son los prestamistas informales, que trabajan fuera de la ley, resulta ilustrativo observar la figura No.2). Donde apreciamos que en la economía mixta los sistemas formales de financiamiento no alcanzan totalmente en su accesibilidad al micro-empresario y los emprendedoresambulantes-autónomos, quienes tienen literalmente como fuente de financiamiento directo a los prestamistas-usureros como prestamista más cercano, debido a la falta de suficiente accesibilidad de las instituciones de micro-finanzas (IMF's), que son técnicamente la fuente oficial de asistencia financiera, a este sector de la economía informal.

Resulta interesante la reflexión que se hace referente a esta práctica en la biblia "el que no da su dinero con usura ni acepta soborno contra el inocente. El que hace estas cosas no resbalará jamás". (Biblia. N.T. Salmos 15: 5) en sentido figurado se explica que la usura es contraria a la práctica cristiana entendida como pecado.

Se hace necesario hacer la distinción entre prestamistas informales: [normales" y "usureros]. Los primeros cobran un interés uniforme, que puede ser desmedido (180\% al año en el caso se estudia) pero se hace con normas conocidas y estándar. El usurero carga intereses en función de la capacidad máxima de pago de cada cliente, llegando a fijar cantidades que no le permitirán nunca pagar el principal, pero sí ir pagando los intereses.

V) Instituciones públicas y privadas de ayuda:

a) Las organizaciones no gubernamentales (ONG's):

Estas entidades están fundamentalmente representadas por organizaciones jurídicas denominadas fundaciones, cuyo rol es canalizar recursos que reciben de patrocinadores, benefactores, filántropos provenientes de las empresas privadas, que donan parte de sus ganancias a estas instituciones de ayuda y servicios a la comunidad, que cumplen diversos roles, para contribuir con la responsabilidad social corporativa (RSC), y financian programas o proyectos de apoyo a los pobres en sus diversas necesidades como: 


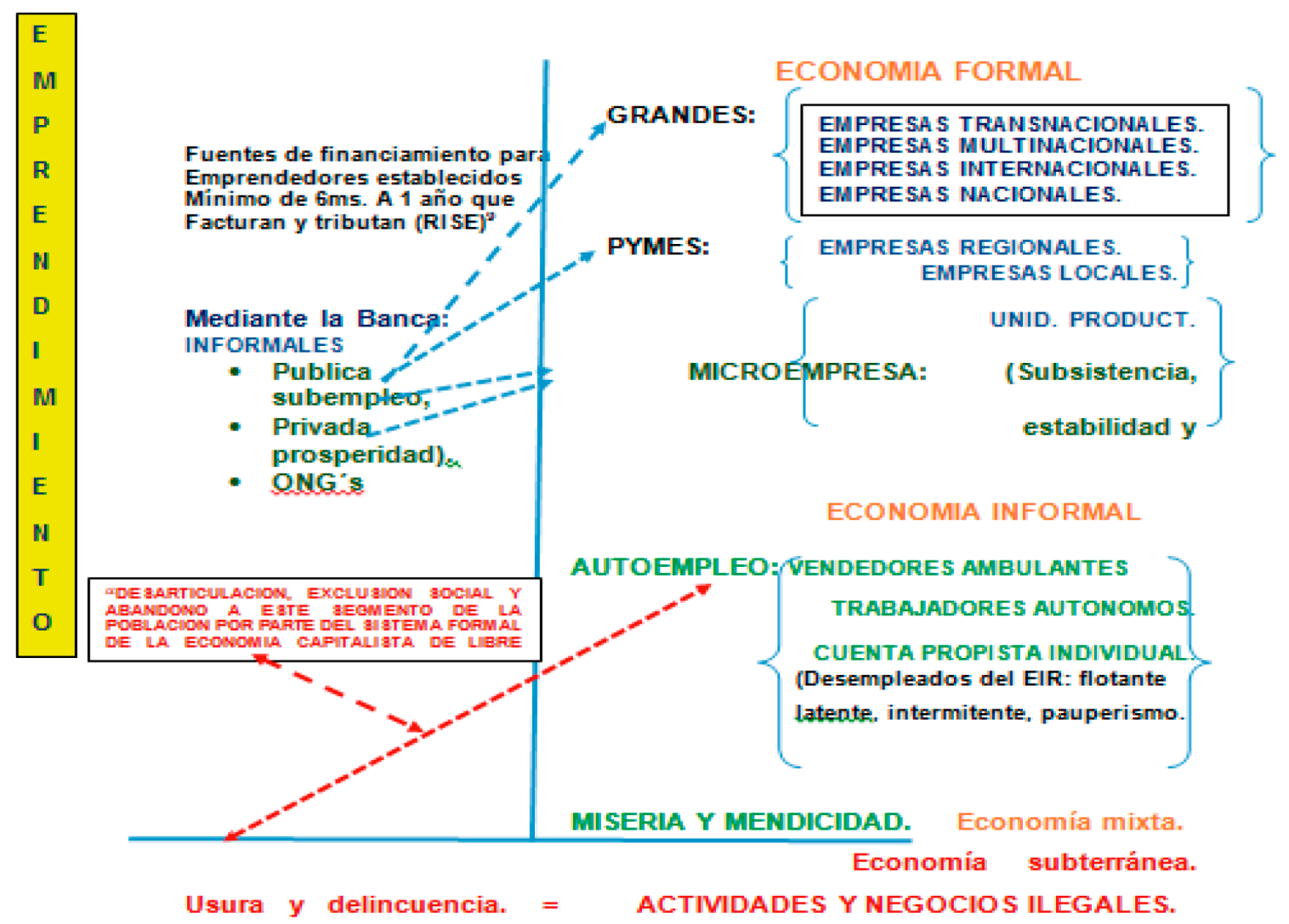

Figura 2. Evolución del emprendimiento en una economía mixta

Fuente: J. Humberto Mancero Mosquera - Elaboración Propia

salud preventiva, vacunación, donaciones de ropa y comida, becas de estudios, juguetes, entre sus diversas actividades.

Además, trabajan en programas vinculados a la construcción de alberges, centros de cuidados para ancianos, huérfanos, escuelas de formación de oficios y talleres para dotar de habilidades y competencias laborales a los más pobres de la sociedad. En ese campo surgieron las ONG's que iniciaron junto a las iglesias, prestamos pequeños para fomentar la iniciativa del emprendimiento proporcionando microcréditos, sin intereses o con bajísimos costos financieros, en plazos cortos, con pagos de frecuencia semanal. Como mecanismo de ayuda para rescatar a los pobres de la usura, buscando un sistema de micro-finanzas para buscar la accesibilidad de apoyo financiero a los más pobres, que desean emprender un micro-negocio en especial de fabricación de artesanías, ropa, alimentos o comercio simple.

La convergencia y la sinergia que se logra mediante el trabajo en redes.

Esta teoría busca lograr que mediante la articulación, integración alrededor de todos los actores sociales, vinculados a contribuir en la solución de reducir la exclusión, inequidad, pobreza y falta de oportunidad para prosperar, mediante el derecho al trabajo digno. Las familias aban- donen la miseria y pobreza extrema, llegando a vivir con dignidad en la sociedad.

Caso Ecuador: Ciudad de Guayaquil, para lograr este anhelado sueño que solo sería posible si se consolidad en un solo proyecto la convergencia de instituciones como las siguientes:

1) Municipio de la ciudad

2) Gobernación del Guayas

3) Instituto de economía popular y solidaria

4) Universidades

5) Instituciones formales de micro-finanzas

6) ONG's

7) Instituto de registros de marcas y patentes

8) institutos de higiene

9) La cámara de microempresas y programas asociativos

10) Instituto ecuatoriano de seguridad social (IESS)

11) Servicios de Rentas internas (SRI) y el régimen impositivo simplificado ecuatoriano (RISE)

Solamente formando la integración de la red social de ayuda al proceso de inclusión social, para los pobres y emprendedores autónomos urbanos y rurales, se podrá ofrecer soluciones reales con impactos de largo plazo. 
Ya se ha experimentado mediantes programas individuales que los diferentes actores sociales han impulsado como iniciativa propia. Toda ayuda es positiva, buena y ayuda, lamentablemente al final terminan siendo soluciones parches, temporales y de impacto en el corto plazo. Porque los cambios gubernamentales, del entorno de la sociedad, no se logran mantener con las innovaciones requeridas de forma permanente.

Al trabajar cada institución por su cuenta, de las once ya mencionadas los resultados beneficiosos se dispersan, no logrando la sinergia que se busca en esta propuesta. Se podría aclarar esta visión prospectiva, utilizando un tradicional algoritmo que ilustra como la convergencia y la sinergia juntas trabajan: $2+2=4$ ya no sería así; porque la convergencia potencializa los resultados de forma tal que se puede lograr el resultado, un resultado mucho mayor como el siguiente: $2+2=6$. Como lograr este aparente milagro, La respuesta se encuentra en la esta simple reflexión: ¿Que es más fácil romper una o dos cuerdas juntas o romper diez cuerdas o más juntas?

La integración y articulación de una "red social con estos actores sociales claves, cumpliendo eficientemente cada uno de sus roles protagónicos, enlazados mediantes objetivos comunes, vinculados y cubiertos con un marco jurídico integrado común a todos quienes que deben participar en esta propuesta, construyen soluciones más fuertes, potenciando los resultados a lograr, con impacto de largo plazo positivos. Debido al acompañamiento durante mínimo 5años, que se debería realizar a cada persona o familia que forma parte del programa de ayuda integrada en la red.

Es fundamental recoger experiencias como las siguientes: integrar la educación, la salud, la aportación y beneficios de la seguridad social, la cultura del ahorro, el desarrollo de buenas prácticas de manufactura, comercio, servicio al cliente, microcrédito, tarjeta de débito, acceso a las redes bancarias, tan solo por mencionar las facilidades sociales más básicas de un ciudadano común. Mediantes las aproximaciones que se realizan en los congresos de microfinanzas, microcrédito y de economía social y estudios más cercanos de las reales necesidades que tienen los sujetos de esta investigación, porque los conocimientos cada vez avanzan y se puede identificar de mejorar forma las aspiraciones reales y comprender, que este grupo objetivo social de estudio; no tiene acceso formal a la educación, becas de estudio, salud pública, jubilación, crédito de vivienda, seguridad social, no paga impuestos, no se capacita organizadamente, no desarrolla habilidades empresariales y comerciales, para competir de mejor forma en su mercado de micro-negocios.

El principal filtro social lo constituyen las policías metropolitanas y publica, que consideran y tratan a los vendedores ambulantes en especial o trabajadores autónomos individuales, como, casi delincuentes: la pregunta es simple: ¿Por qué?:
1) No tiene los permisos de uso de suelos para ocupar la vía pública con sus emprendimientos.

2) No tienen las patentes de funcionamiento de sus locales.

3) No poseen registros sanitarios de sus productos.

4) No tienen marca registrada de sus productos.

5) No aplican necesariamente de forma visible, buenas prácticas de manufactura,

6) No es confiable siempre el origen de los insumos de materias primas que utilizan.

7) No siempre se identifica el origen de los productos, que comercializan públicamente, rondando el fantasma, de que sean productos robados o de contrabando.

8) No tienen una buena imagen física en higiene, asepsia y presentación física de ellos mismos como trabajadores organizados y autorizados. Para ofrecer seguridad y confianza a sus clientes.

Como se puede apreciar las razones son altamente justificadas, la solución ya no va por la vía de la sanción, castigo, represión, al contrario se deben buscar soluciones creativas diferentes innovadoras, distintas para integrar, incluir socialmente a este productivo sector, que solo cometió en inicio a su actividad el gran delito, de inventarse un trabajo, porque no lo encontró o en sociedad lo que le pagaban, eran muy bajo.

\section{Conclusiones}

- Diferenciar objetivamente quienes deben ser las instituciones que participen como proveedores de servicios integrados, para potencializar sinérgicamente las micro-finanzas, para que robustecidas de forma convergentes, con los aportes los demás actores sociales, se articulen sus objetivos y políticas públicas, al mismo gran objetivo esencial: liberar del circulo de la miseria y pobreza, para llevar al nivel de la prosperidad y dignidad en el largo plazo, a los micro-emprendedores fijos y autónomos ambulantes.

- Reducir las prácticas de la usura en la ciudad y el país.

- Fomentar el surgimiento de más institución con visión integral de lo que son realmente las micro-finanzas y su impacto directo en el desarrollo del sector informal de la economía, buscando el impacto de resultados eficientes en el largo plazo y articulando en el corto plazo, las soluciones intermedias para cumplir la transición dependiendo de las condiciones y grados de necesidad, que requieren los sujetos de estudio a quienes se busca ayudar.

- Cada vez se evidencia la necesidad de articular de mejor formar, como las instituciones del sector público, y privado, para aplicar de adecuadas formas los postulados de la "Responsabilidad Social Empresarial o Corporativa", logren en conjunto servir, ayudar, integrar socialmente a los pobres y micro-emprendedores informales de la ciudad y el país. 


\section{Recomendaciones}

- Implementar la propuesta como solución innovadora optimizando ya experiencias existentes como: las alianzas estratégicas empresariales, los modelos de asociatividad y agremiación micro-empresarial institucional.

- Lograr articular la constitución en esta gran red, para consolidar de forma más efectiva, eficaz y eficiente a todos los actores sociales claves. Orientados al liberar de la usura, la exclusión e inequidad que viven los pobres y micro-emprendedores, fijos y ambulantes autónomos de Guayaquil - Ecuador. Pudiéndose replicar la experiencia en otras provincias del país.

\section{Referencias Bibliográficas}

Armendariz, B., y Morduch, J. (2010). The economics of microfinance (2nd ed ed.). MIT Press.

CGAP. (2009). Financial access 2009. medición del acceso a los servicios financieros alrededor del mundo. Descargado de https://www.cgap.org/ sites/default/files/CGAP-Financial -Access-2009-Spanish.pdf

Collins, D., Morduch, J., Rutherford, S., y Ruthven, O. (2009). Portfolios of the poor: how the world's poor live on $\$ 2$ a day. Princeton University Press. Descargado de http: / / public.eblib.com/choice/ publicfullrecord.aspx?p=457710 (OCLC: 439844627)

Contreras Soto, R. (2007). Necesidades del comercio al por menor en celaya: pequeños y micro negocios. Eumed.net. Descargado de http: / / site.ebrary .com/id/10576618 (OCLC: 811371753)

Flores, R. M. (2005). El microcrédito en el seno del grameen bank. , 13-14.

Harrison, T. S., y Thompson, N. W. (1975-08). Multiple endocrine adenomatosis-i and II. , 1-51.

Jansson, T. (2001). Microfinance: From village to wall street?

Kurt Koenigsfest. (2017). Nuestros clientes. Descargado de https://www.bancosol.com.bo/ quienes-somos/nuestros-clientes

Lacalle Calderón, M. (2001). Los microcréditos : un nuevo instrumento de financiación para luchar contra la pobreza. Descargado de http: / / hdl . handle . net/10272/394

León Guzmán, M. (2013). Sector y empleo informales en ecuador. Descargado de http: //www.eltelegrafo.com.ec/ noticias/masqmenos/1/sector-y -empleo-informales-en-ecuador

Munoz-Torres, M. J., Fernandez-Izquierdo, M. A., y Balaguer-Franch, M. R. (2004-07). The social responsibility performance of ethical and solidarity funds: an approach to the case of spain. , 13(2), 200218. Descargado de http://doi.wiley.com/
$10.1111 / j .1467-8608.2004 .00363 . x$ doi: $10.1111 / \mathrm{j} .1467-8608.2004 .00363 . x$

Nair, A. (2005-02-08). Sustainability of microfinance self help groups in india: Would federating help? The World Bank. Descargado de http: / / elibrary.worldbank.org/doi/ book/10.1596/1813-9450-3516 (DOI: $10.1596 / 1813-9450-3516)$

Natalí Vargas. (2016). ¿qué dice harvard sobre BancoSol? - diario pagina siete. Descargado de http://www.paginasiete.bo/ inversion/2016/4/17/que-dice -harvard-sobre-bancosol-93430.html

Reina, C. d., y Valera, C. d. (2012). Santa biblia: antiguo y nuevo testamento : antigua versión de casiodoro de reina (1569), revisada por cipriano de valera (1602) y cotejada posteriormente con diversas traducciones, y con los textos hebreo y griego. Editorial Vida. (OCLC: 796763637)

Serrano Mancilla, A. (2010). Análisis de informalidad en ecuador: recetas tributarias para su gestión.

World Bank. (2007-11-20). Finance for all?: Policies and pitfalls in expanding access. The World Bank. Descargado de http:// elibrary . worldbank.org/doi/book/ $10.1596 / 978-0-8213-7291-3$ 10.1596/978-0-8213-7291-3)

Yunus, M. (2011). ¿es posible acabar con la pobreza? Editorial Complutense. (OCLC: 912454349)

Recibido: 5 de agosto de 2017 Aceptado: 13 de septiembre de 2017 